\title{
Exploring the limits of soft $x$-ray magnetic holography: Imaging magnetization reversal of buried interfaces (invited)
}

\author{
J. Camarero, ${ }^{1,2, a)}$ E. Jiménez, ${ }^{1}$ J. Vogel, ${ }^{3}$ C. Tieg, ${ }^{4, b)}$ P. Perna, ${ }^{2}$ A. Bollero, ${ }^{2}$ \\ F. Yakhou-Harris, ${ }^{4}$ C. Arm, ${ }^{5}$ B. Rodmacq, ${ }^{5}$ E. Gautier, ${ }^{5}$ S. Auffret, ${ }^{5}$ B. Delaup, ${ }^{5}$ \\ G. Gaudin, ${ }^{5}$ B. Dieny, ${ }^{5}$ and R. Miranda ${ }^{1,2}$ \\ ${ }^{1}$ Departamento de Física de la Materia Condensada and Instituto "Nicolás Cabrera", Universidad Autónoma \\ de Madrid, 28049 Madrid, Spain \\ ${ }^{2}$ Instituto Madrileño de Estudios Avanzados en Nanociencia IMDEA-Nanociencia, Campus Universidad \\ Autónoma de Madrid, 28049 Madrid, Spain \\ ${ }^{3}$ Institut Néel, CNRS and UJF, BP 166, F-38042 Grenoble, France \\ ${ }^{4}$ European Synchrotron Radiation Facility ESRF, BP 220, 38043 Grenoble, France \\ ${ }^{5}$ SPINTEC, CEA/CNRS/UJF/INPG, INAC-CEA, F-38054 Grenoble, France
}

(Presented 15 November 2010; received 14 October 2010; accepted 6 January 2011; published online 15 April 2011)

\begin{abstract}
Only a very few experimental techniques can address the microscopic magnetization reversal behavior of the different magnetic layers in a multilayered system with element selectivity. We present an element-selective study of ferromagnetic $(\mathrm{FM})[\mathrm{Co} / \mathrm{Pt}]_{\mathrm{n}}$ multilayers with perpendicular anisotropy exchange-coupled to antiferromagnetic (AFM) FeMn and IrMn films performed with a new experimental set-up developed for both soft x-ray spectroscopy and holography imaging purposes. The spectroscopy analysis allows the quantification of the unpinned (pinned) uncompensated AFM moments, providing direct evidence of its parallel (antiparallel) alignment with respect to the FM moments. The holography experiments give a direct view of both FM and uncompensated AFM magnetic structures, showing that they replicate to each other during magnetization reversal. Remarkably, we show magnetic images for effective thicknesses as small as one monolayer. Our results provide new microscopic insights into the exchange coupling phenomena and explore the sensitivity limits of these techniques. Future trends are also discussed. (C) 2011 American Institute of Physics. [doi:10.1063/1.3567035]
\end{abstract}

\section{INTRODUCTION}

Dimensionality and interfacial effects promote much of the new properties observed in complex multilayered magnetic nanostructures. ${ }^{1}$ Knowledge of the magnetic structure at the interfaces during magnetization reversal is hence essential for a better understanding and control of these complex systems. The development of techniques that have the potential of analyzing magnetic properties with nanometer spatial resolution has contributed essentially to our present level of understanding of micromagnetic phenomena. ${ }^{2}$ However, the lack of techniques capable of providing detailed magnetic information of buried interfacial layers with element selectivity and upon external fields is delaying this understanding.

Polarized synchrotron radiation has recently given rise to innovative possibilities for imaging magnetic materials with nanometer resolution. ${ }^{3}$ Scattering based techniques, such as soft $\mathrm{x}$-ray magnetic resonant scattering ${ }^{4}$ and lensless holographic imaging, ${ }^{5}$ have been established as powerful tools for studying magnetic structures in surfaces and thin films with element selectivity on the nanometer length scale. The soft $\mathrm{x}$-ray range hosts the largest magnetic resonances of the magnetically important transition-metal and rare-earth series. Additionally, holographic imaging combines the magnetic sensitivity obtained with $\mathrm{x}$-ray magnetic circular

\footnotetext{
${ }^{a)}$ Electronic mail: julio.camarero@uam.es.

${ }^{\text {b) }}$ Present address: Helmholtz-Zentrum Berlin, D-12489 Berlin, Germany.
}

dichroism in transmission geometry with the spatial resolution from a simple Fourier inversion of a reciprocal space soft x-ray interference pattern from an object (sample) and aerence aperture (which defines the final spatial resolution). In addition, the technique can image deeply buried magnetic systems and, as a pure photon-based technique (photon-in/ photon-out), can be used in applied magnetic fields. Up to now, soft x-ray holography has focused on [nonmagnetic/ magnetic $]_{n}$ multilayers with perpendicular magnetic anisotropy ${ }^{5-10}$ using Pt (Refs. 5-7, 10) and Pd (Refs. 8 and 9) as nonmagnetic layers, and Co (Refs. 5-8, 10) and CoNi (Ref. $8)$ as magnetic layers, with relatively large $(5-60 \mathrm{~nm})$ effective magnetic thicknesses for both remanence ${ }^{5-8,10}$ and fielddependent ${ }^{6,9,10}$ measurements.

We have recently spread out the capabilities of soft x-ray holography for imaging the magnetization reversal in thin magnetic films, by adding the quantitative aspects of magnetic spectroscopy. ${ }^{11}$ We have investigated ferromagnetic (FM) $[\mathrm{Co} / \mathrm{Pt}]_{\mathrm{n}}$ multilayers with perpendicular anisotropy exchange coupled with antiferromagnetic (AFM) FeMn and IrMn films. Macroscopically both FM/FeMn and FM/IrMn systems behave similarly, but different transmission sensitivities have been found for them regarding to explore the capability to image the uncompensated AFM domain structure. The motivation for this study is to address the magnetization reversal processes of perpendicular exchange-biased systems as well as to explore the sensitivity limits of the technique. 
The paper is organized as follows. The experimental details are described in Sec. II. The exchange coupling phenomenon is briefly explained in Sec. III. The spectroscopic and imaging measurements are discussed in Secs. IV and V, respectively. The conclusions and future trends are discussed in Sec. VI.

\section{EXPERIMENTAL}

The experiments were carried out at beamline ID08 of the European Synchrotron Radiation Facility (ESRF) which provides $100 \%$ circularly polarized light in the soft $x$-ray region. The experimental layout and the sample-mask structure are schematically shown in Fig. 1. Our set-up enables both quantifying magnetic properties of the whole system by spectroscopic means and imaging magnetic domains with element selectivity and in external applied fields.

The sample-mask structures were prepared by sputtering $[1.8 \mathrm{~nm} \mathrm{Pt} / 0.6 \mathrm{~nm} \mathrm{Co}]_{\mathrm{n}} / \mathrm{AFM}$, with $\mathrm{n}=8$ and 4, onto a 100 $\mathrm{nm}$ thick $\mathrm{Si}_{3} \mathrm{~N}_{4}$ membrane, which was precovered with a 1.2 $\mathrm{nm}$ Pt buffer layer. Different AFM layers have been used, $10 \mathrm{~nm}$ FeMn and $5 \mathrm{~nm}$ IrMn. The AFM film was then capped with $3 \mathrm{~nm} \mathrm{Al}$ to prevent oxidation. The back-side of the membrane supports an opaque $[100 \mathrm{~nm} \mathrm{Au} / 10 \mathrm{~nm} \mathrm{Cr}]_{10}$ multilayer with a $1.8 \mu \mathrm{m}$ hole). The latter defines the field-ofview (FOV) of the magnetic image. The $1.1 \mu \mathrm{m}$ thick [Au/ $\mathrm{Cr}$ ] mask guarantees that the $\mathrm{x}$-ray beam is transmitted only through the object and reference holes. Five smaller apertures around the object hole, each guiding a reference beam, ${ }^{12}$ were milled through the whole structure, as shows the inset of Fig. 2. The diameter of the reference holes is in the range of 100-330 nm, and the distance to the object aperture is $3.7 \mu \mathrm{m}$, smaller than the transverse coherence length of the light $(\xi)$. The latter depends on the wavelength of the photons $(\lambda)$, the size of the source $(d)$ and its distance from the sample $(D)$. Here we placed a $50 \mu \mathrm{m}$ diameter pinhole (secondary source) at $1.5 \mathrm{~m}$ from the sample. Then, at the Co- $L_{3}$ absorption edge, the transverse coherence is $\xi=\lambda D / 2 \pi d>7 \mu \mathrm{m}$.

(a)

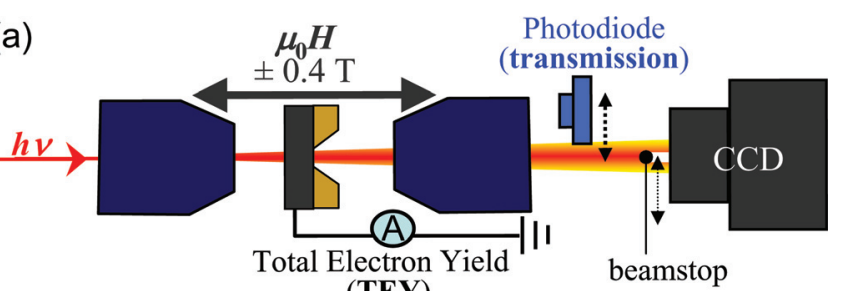

(b)

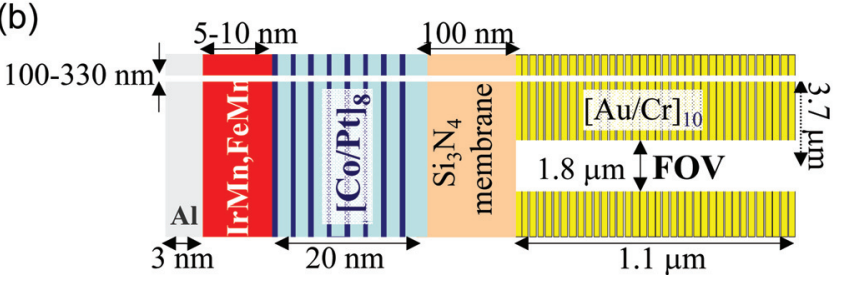

FIG. 1. (Color online) (a) Scheme of the experimental layout developed for spectroscopy and imaging purposes. (b) Sample-mask structure cross section showing the aperture for the field-of-view (FOV) and one aperture for the reference beam.

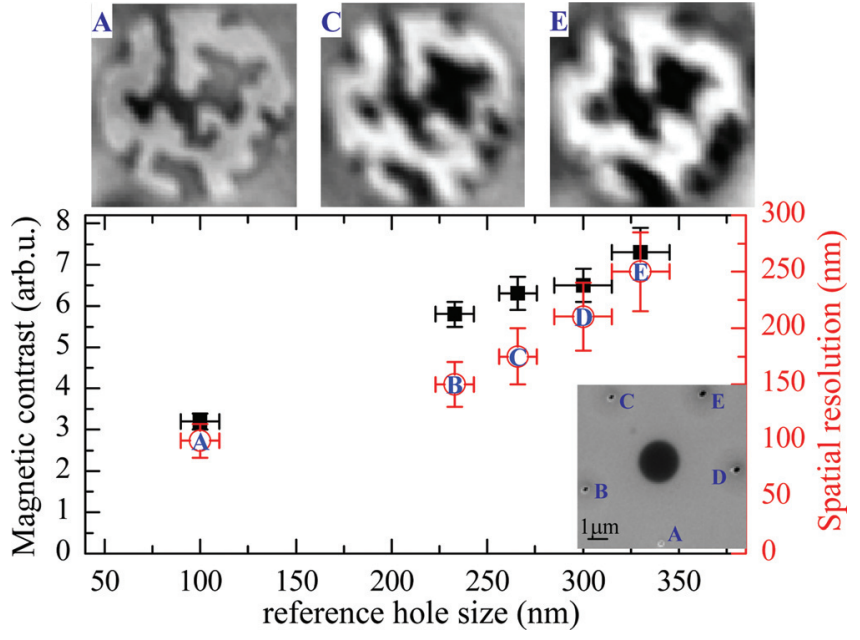

FIG. 2. (Color online) Effect of reference hole size on domain contrast (squares) and spatial resolution (circles) extracted from magnetic images (top) of a demagnetized $[\mathrm{Pt} / \mathrm{Co}]_{8} / 10 \mathrm{~nm}$ FeMn sample. The magnetic contrast is taken from the difference between the two prominent gray scale values of the magnetic images, which correspond to Co domains with opposite out-ofplane magnetization, whereas the resolution is given by the domain wall width from line scans taken through opposite magnetic domains. The reference hole size is estimated from the scanning electron microscopy image taken from the mask-side of the structure shown in the bottom right inset.

The sample-mask structures were field-cooled in a +0.2 $\mathrm{T}$ perpendicular magnetic field and mounted in a highvacuum chamber. The $\mathrm{x}$-ray beam was incident along the surface normal with the AFM films facing the beam. An electromagnet mounted outside allows to apply external magnetic fields perpendicular to the film surface, i.e., parallel to the x-ray beam. Both, x-ray absorption and holography measurements were performed at room temperature (RT). The absorption measurements were obtained by recording simultaneously the total electron yield (TEY), from the sample photocurrent measured with a picoamperemeter, and the transmitted photon intensity, by using a movable photodiode (see Fig. 1).

Holograms were acquired for opposite photon helicities at the $L_{3}$ absorption edges. The direct transmitted beam was blocked by a beam stop of $0.5 \mathrm{~mm}$ diameter and the holograms were recorded by a 16-bit back-illuminated chargecoupled device $(C C D)$, with $1300 \times 1340$ pixels, placed at a distance of $45 \mathrm{~cm}$ from the sample-mask position. The intensity of the hologram carries the phase relation between object and reference beam. The real-space image of the sample structure is reconstructed by a fast Fourier transform (FFT) of the hologram in real-time during the experiment. Magnetic contrast is achieved by taking the difference between holograms recorded with opposite photon helicity at the $L_{3}$ absorption edge. Direct Fourier inversion of the difference pattern, i.e., magnetic hologram, yields an image of the magnetic domain structure within the object hole. ${ }^{5}$ The total acquisition time used to get the magnetic hologram depends on the total amount of dichroism signal to resolve in real space and on the type of measurement, i.e., static or during magnetization reversal. For example, for imaging a FM layer with an effective thickness of about $5 \mathrm{~nm}$, total acquisition times of about 4 min were employed for static measurements (e.g. see 
Fig. 2), including the acquisition of several frames per photon helicity and the helicity change. Shorter acquisition times were used for imaging the magnetization reversal, in order to avoid magnetic relaxation processes during the acquisition.

The quality of the magnetic image is given by both magnetic contrast and spatial resolution. To achieve a reasonable contrast in the reconstructed image, the intensity of the reference beam should be comparable to the object beam. For example, for a typical mask with object and reference hole diameters of about $1.8 \mu \mathrm{m}$ and $200 \mathrm{~nm}$, respectively, the intensity ratio of object and reference beam before traversing the sample is 80:1. Taking into account the absorption by the membrane and the sample structure, the intensity transmitted through the object beam is reduced and the ratio is slightly lowered. For example, this gives an absorption-corrected intensity ratio of 50:1 at the Co- $L_{3}$ absorption edge. In addition, though the ultimate spatial resolution of the reconstructed image is in principle limited by the wavelength, the resolution of the reconstructed image in real space is given by the size of the reference pinhole. ${ }^{5}$ In practice, the image is as if it was painted by a pencil of tip similar to the size of the reference hole.

Figure 2 illustrates the aforementioned effects of reference hole size on magnetic contrast and resolution. The magnetic images shown on top were retrieved from the magnetic hologram acquired at Co- $L_{3}$ absorption edge of a samplemask structure with five reference holes of different size. For instance, the image retrieved from the largest reference aperture shows higher magnetic contrast but blurred domain structure (top right image). In general, the contrast increases with the reference hole size while resolution diminishes.

\section{FM/AFM EXCHANGE COUPLING PHENOMENA}

The exchange coupling between ferromagnetic (FM) and antiferromagnetic (AFM) layers remains among the most intriguing fundamental interfacial magnetic phenomena and it is at the trend of today (and future) applications in many areas, such as magnetic recording, sensors and actuators, spintronics, or biomedicine. Particularly important is the unidirectional coupling between the AFM and FM layers, referred to as exchange bias. ${ }^{13}$ This phenomenon is actually used in advanced magnetic in-plane spintronic devices ${ }^{14}$ and the future advances are promoted by the recent observation of room temperature perpendicular exchange-coupling effects in several FM/AFM systems. ${ }^{15}$ The underlying physics of the exchange bias was already correctly described almost half a century ago by the original work of Meiklejohn and Bean. ${ }^{13}$ Upon cooling a FM/AFM system in an applied magnetic field to below the Néel temperature of the AFM layer, uncompensated spins at the AFM surface align with the polarized FM interface. Although the exchange interaction is strongly reduced, i.e., up to two orders of magnitude, AFM materials are used to pin (or stabilize) the magnetization direction of a FM reference layer. Among others, coercivity enhancement, ${ }^{14}$ and asymmetric magnetization reversal, ${ }^{16,17}$ are associated phenomena usually observed in exchange-coupled FM/AFM systems.

Recent advances in soft $\mathrm{x}$-ray magnetic-sensitive XMCD-based experimental techniques have thrown light on the basic mechanisms that explain some unusual properties observed in these systems. For example, only a small fraction of uncompensated AFM spins contribute in real FM/ AFM systems (which have unavoidable rough interfaces), unlike for a perfectly flat interface, which explains the rather small size of the exchange bias field $\mu_{0} H_{E}$. In addition, it has already been found that pinned (unpinned) uncompensated AFM spins at the interface are correlated with the exchange bias ${ }^{18}$ (coercivity enhancement). ${ }^{19}$ These features have been addressed in both in-plane and perpendicular ${ }^{20}$ exchange coupled FM/AFM systems. However, a microscopic imaging of most of the aforementioned effects during magnetization reversal, i.e., in external magnetic fields, is still lacking.

\section{SPECTROSCOPY ANALYSIS}

The analysis of the soft $\mathrm{x}$-ray absorption spectra collected simultaneously by recording both the total electron yield (TEY) and the transmitted photon intensity, provides valuable (quantified) information about the magnetic properties of the exchange-coupled FM/AFM systems, about their depth location, as well as about the possibility to exploit the dichroism in the transmitted beam intensity for imaging.

Element-selective magnetic quantification of the FM/ AFM systems can be achieved from XMCD spectra (see Fig. 3 and Fig. 4) and from XMCD element-selective hysteresis loops recorded at selected $L_{3}$ absorption edges (central panel in Fig. 6). The latter shows a coercive field $\mu_{0} H_{C}=55 \mathrm{mT}$, enhanced with respect to a single FM system (of about 2 $\mathrm{mT})$, and a clear nonzero exchange bias field $\mu_{0} H_{E}=-6$ mT. Both are usual effects of FM/AFM systems.

Figure 3 shows raw XAS spectra (top) at the $\mathrm{Fe}$ and Co $L_{2,3}$ absorption edges of the $[\mathrm{Pt} / \mathrm{Co}]_{8} / 10 \mathrm{~nm}$ FeMn sample recorded at negative saturation with both positive and negative photon helicities, and the corresponding XMCD spectra (bottom). The obvious difference between the absorption spectra can be understood by considering the different information depths of both detection modes. In the TEY detection

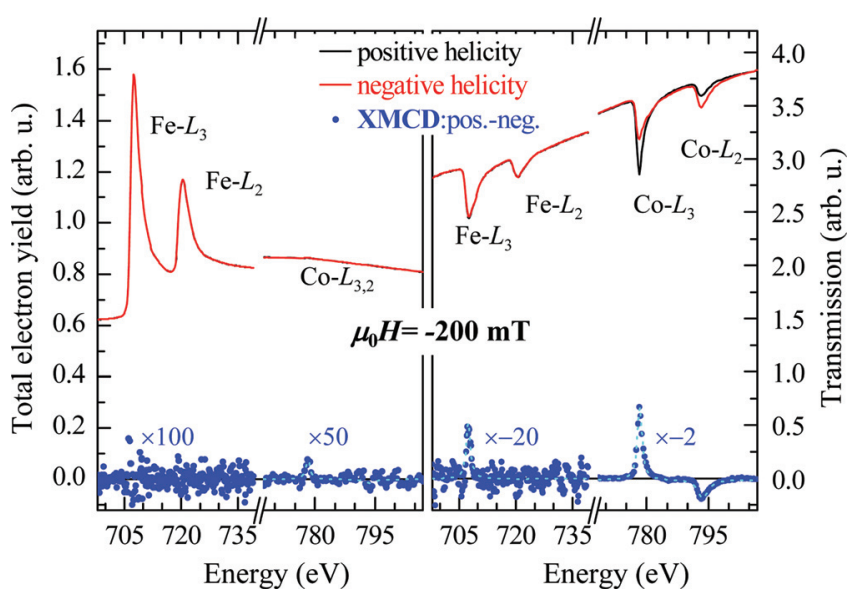

FIG. 3. (Color online) Absorption and XMCD spectra at the Fe- $L$ and Co- $L$ edges of a $[\mathrm{Pt} / \mathrm{Co}]_{8} / 10 \mathrm{~nm}$ FeMn sample recorded by the detection of the TEY signal (left) and the transmitted photon intensity (right). The sample is placed with the FeMn layer facing the incoming x-ray beam in a external field of $\mu_{0} H=-200 \mathrm{mT}$. The $\mathrm{x}$-axis of the graphs is split for clarity. The spectral differences in both detection modes result from different probing depth and different background contributions (see text). Note that the small dichroism signal at the Fe- $L$ edges is only seen by transmission detection. 
mode the information depth is limited to about $2 \mathrm{~nm}$, which corresponds to the electron escape depth. ${ }^{21}$ On the other hand, the photon penetration length is about 50 times larger at the absorption edges. Thus, TEY measurements are more sensitive to the topmost part of the FeMn layer while transmission measurements probe the whole system. The surface sensitivity of the TEY detection explains the larger $\mathrm{Fe}$ absorption signal in comparison to the Co absorption signal. The difference in probing depth is also the origin of the larger absorption Co signal in the transmission spectra.

Similar arguments can be used to explain the differences between XMCD spectra. Larger Co-XMCD signal is found in transmission because is integrated over the whole buried FM layer. In turns, a clear dichroism signal was found at the $\mathrm{Fe}$ $L_{2,3}$ absorption edges in transmission mode, while vanished in the TEY mode. This indicates that the moments contributing to this signal, i.e., referred as uncompensated AFM moments, are not homogeneously distributed over the AFM layer but deeply buried from the top AFM surface. In addition, the same sign of both Fe and Co XMCD signals reveals a parallel alignment between the FM and the net (uncompensated) AFM moments. The normalized XMCD signal measured in transmission at the Fe- $L_{3}$ and Co- $L_{3}$ edge corresponds to 2 and $60 \%$ of the polarization-averaged absorption signal, respectively. By using sum rule analysis, we estimate an effective thickness of uncompensated Fe moments of ( $1.6 \pm 0.5)$ monolayers (ML) assuming that they are localized at the AFM/FM interface, which is strongly supported by the vanishing TEYXMCD signal. Similar values were found in perpendicular exchange-biased FM/FeMn (Ref. 20) and FM/IrMn (Ref. 11) systems, and smaller values in in-plane exchange-biased FM/ IrMn systems $(\sim 0.5 \mathrm{ML})$. $^{22}$

Similar measurements have been performed in a $[\mathrm{Pt} / \mathrm{Co}]$ ${ }_{8} / 5 \mathrm{~nm}$ IrMn sample. In this case, a small but still detectable Mn-XMCD signal in the TEY mode was found, but vanishing in transmission mode (see Fig. 3 of the supplementary information given in Ref. 11). Figure 4 shows the TEYXMCD spectra acquired at the $\mathrm{Mn}-L_{2,3}$ edges at positive and negative saturation fields. The clear Mn-XMCD reveals the presence of uncompensated Mn moments, and the different dichroic values at positive and negative saturation fields suggest the existence of two types of uncompensated AFM

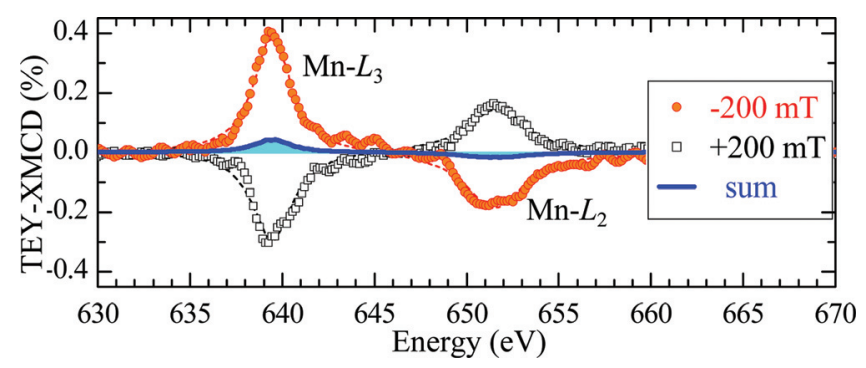

FIG. 4. (Color online) Normalized TEY-XMCD spectra acquired at the Mn$L_{2,3}$ edges in negative (filled circles) and positive (empty squares) saturation of a $[\mathrm{Pt} / \mathrm{Co}]_{8} / 5 \mathrm{~nm}$ IrMn sample. The solid line is the sum of the averaged $\mathrm{XMCD}$ spectra. The clear nonzero sum spectra at the $\mathrm{Mn}-L$ edge is related to the amount of uncompensated AFM moments which are fixed (pinned) during FM reversal, and its positive sign indicates that they are oriented opposite to the field cooling direction. moments. While some uncompensated AFM moments rotate during FM reversal (i.e., they are unpinned), a few of them are fixed (pinned). From the magnitude of the sum curve we quantify that only $10 \%$ of the uncompensated AFM moments are pinned, and from its sign we conclude that the pinned moments are oriented in the direction of the exchange bias field, i.e., opposite to the field cooling direction. This indicates a preferred antiparallel alignment between the FM and the pinned AFM moments, which suggests an antiferromagnetic coupling across the interface between them. This direct evidence confirms recent reports of antiparallel (parallel) alignment between the FM and the pinned (unpinned) AFM moments for both in-plane ${ }^{23}$ and perpendicular ${ }^{24}$ exchange biased FM/IrMn systems.

Note that the amount of uncompensated AFM moments derived from the spectroscopy analysis is similar for both $[\mathrm{Pt} / \mathrm{Co}]_{8} / \mathrm{AFM}$ systems and that different detection modes needed to be used, i.e., Fe-XMCD signal is just found in transmission mode for FM/10 nm FeMn whereas MnXMCD signal is just found in the TEY mode for FM/5 nm IrMn. In both cases the top AFM surface was faced to the incoming $\mathrm{x}$-ray beam. This apparent discrepancy can be ascribed to the difference in both probing depths (thinner AFM IrMn layer), photon flux (lower at the Mn edge), and absorption properties (slightly larger at the Mn edge). For instance, the vanishing Fe-XMCD signal in the TEY measurements for the FM/FeMn system indicates that the uncompensated moments are deeply buried, as discussed above. On other hand, the photon intensity passing the reference holes gives a significant offset in the transmission spectra, which makes more difficult to detect tiny dichroic signals related to the uncompensated AFM moments (ca. 1\% of the total absorption). In addition, the size of the reference hole of the FM/IrMn sample-mask structure was 1.5 times larger than the corresponding to $\mathrm{FM} / \mathrm{FeMn}$, which turn out in larger transmitted intensity backgrounds at the absorption edges for the former. All this results in a vanishing Mn-XMCD signal in transmission measurements for the FM/IrMn system. Hence, for the present samples, the possibility to exploit the dichroism in transmission measurements for imaging the domain structure of the uncompensated AFM moments was only able for the FM/FeMn system. (a)

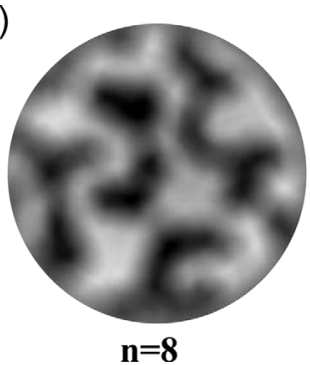

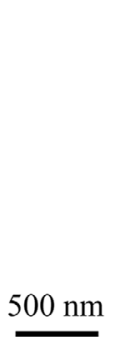

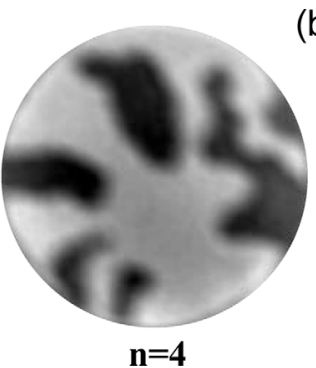

(b)

FIG. 5. Co magnetic domain images of $[\mathrm{Pt} / \mathrm{Co}]_{n} / 5 \mathrm{~nm}$ IrMn films with $\mathrm{n}=8$ (a) and $n=4$ (b) in their demagnetized states. The images are retrieved from the spatial Fourier transformation of magnetic holograms acquired at the Co- $L_{3}$ absorption edge, as described in the text. The derived domain periodicity (reference hole size) are of about $500 \mathrm{~nm}(150 \mathrm{~nm})$ and $700 \mathrm{~nm}(100$ $\mathrm{nm}$ ) for the film with $\mathrm{n}=8$ and $\mathrm{n}=4$, respectively. 


\section{HOLOGRAPHY IMAGING}

The magnetic domain structures can be imaged in any applied magnetic fields with element selectivity by soft $\mathrm{x}$-ray holography measurements, as described above.

Figure 5 compares the magnetic domain structure of the FM layer of $[\mathrm{Pt} / \mathrm{Co}]_{\mathrm{n}} /(5 \mathrm{~nm})$ IrMn samples, with $\mathrm{n}=8$ and 4 which correspond with an equivalent Co thickness of 4.8 and $2.4 \mathrm{~nm}$, respectively, acquired in zero field after demagnetizing the sample in an alternating magnetic field with a decreasing amplitude. The sample with more Co $(n=8)$ shows a more blurred image because the size of its reference hole $(150 \mathrm{~nm})$ is only 3.3 times smaller than its domain periodicity (of about $500 \mathrm{~nm}$ ), whereas the corresponding one to $\mathrm{n}=4(100 \mathrm{~nm})$ is 7 times smaller than its domain periodicity (of about $700 \mathrm{~nm}$ ). The images show random magnetic domain structures with periodicities at least one order of magnitude smaller than in similar perpendicular anisotropy FM systems, as expected for FM/AFM systems. The smaller domain periodicity found for the thicker sample $(n=8)$ has been also found in perpendicular exchange-biased $[\mathrm{Pt} / \mathrm{Co}]_{n} /$ FeMn systems by means of soft $\mathrm{x}$-ray magnetic scattering measurements. ${ }^{20}$ This originates from the low dimensionality and the minimization of dipolar effects in perpendicular anisotropy systems. The domain size is determined by the balance between the magnetostatic energy, which reduces upon domain formation, and the domain wall energy. Hence, the difference in domain size is likely to be due to the larger total magnetization, ${ }^{25}$ and therefore dipolar interaction, for the $\mathrm{n}=8$ sample.

The evolution of the magnetic domain structure of the FM layer along the whole hysteresis loop of the $[\mathrm{Pt} / \mathrm{Co}]_{8} / 5$ $\mathrm{nm}$ IrMn sample has been reported elsewhere. ${ }^{11}$ The images showed that the magnetization reversal is characterized by nucleation of magnetic domains and domain wall propagation processes. Different magnetic domain configurations were also found in the increasing and decreasing field branches of the hysteresis loop, i.e., asymmetric reversal. This showed for the first time that holography experiments allow imaging the magnetization reversal of an exchangebiased FM layer with an equivalent Co thickness below $5 \mathrm{~nm}$ in real space and in external magnetic fields. But, we could not image the magnetic structure of the uncompensated AFM moments because of the vanishing Mn-XMCD signal found in transmission experiments, as discussed above.

The element-selectivity capability of soft x-ray holography has been exploited for the $[\mathrm{Pt} / \mathrm{Co}]_{8} / 10 \mathrm{~nm}$ FeMn sample. Here we show for the first time the magnetic structure of the uncompensated AFM moments during FM reversal. Figure 6 displays element-selective magnetic images taken at positive saturation (right images) and during the decreasing field branch (left) of the hysteresis loop. The small but significant total integrated XMCD signal in transmission at the Fe- $L_{3}$ edge (30 times smaller than the signal at the Co- $L_{3}$ edge, as Fig. 3 shows) implies that long acquisition times had to be used for imaging the uncompensated AFM moments, i.e., fifteen times longer than for the Co images (for which we used $1 \mathrm{~min})$. At positive saturation, the element-selective magnetic images show just bright gray scale values whereas dur-
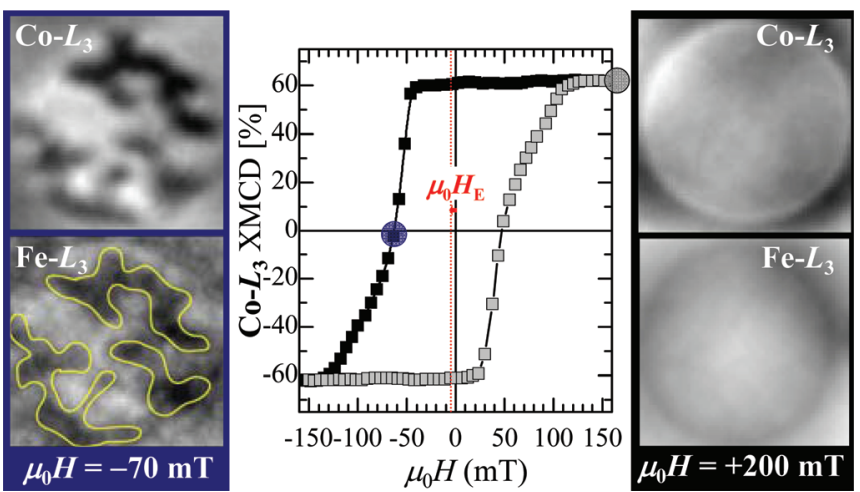

FIG. 6. (Color online) Element-selective magnetic images of the $[\mathrm{Pt} / \mathrm{Co}]_{8} /$ $10 \mathrm{~nm}$ FeMn sample during magnetization reversal. Center: element-selective hysteresis loop recorded in transmission. The images retrieved from magnetic holograms (reference hole $\sim 100 \mathrm{~nm}$ ) recorded at Co- $L_{3}$ (top) and $\mathrm{Fe}-L_{3}$ (bottom) absorption edges represent the magnetic domain structure of the FM layer and the uncompensated AFM moments, respectively. The magnetic contrast of the latter corresponds to an effective thicknesses as small as one monolayer thick, as derived from the spectroscopic analysis (see Fig. 3), and that mimics the FM magnetic domain structure.

ing the decreasing field branch several coincident magnetic domains (black) are observed. This reveals that the uncompensated AFM moments replicate the magnetic domain structure of the FM layer, proving that the FM moments locally drag the unpinned AFM moments during magnetization reversal. The energy cost to do that would explain the coercivity enhancement found in FM/AFM systems. Thus, the holography imaging experiments allow visualizing the magnetization reversal processes and the direct correlation between the magnetic structure of both FM and unpinned AFM moments (less than $1 \mathrm{ML}$ ) in real space and in external magnetic fields.

Unfortunately, the total integrated dichroism signal of the pinned AFM moments was really small, i.e., effective AFM thickness of $0.1 \mathrm{ML}$ (see Fig. 4), meaning that we could not identify (image) where they were located.

\section{CONCLUSIONS AND FUTURE TRENDS}

We have explored the unique capabilities of soft $\mathrm{x}$-ray spectroscopy and holography measurements for quantifying and imaging the magnetization reversal of magnetic complex systems with element (layer) selectivity, spatial resolution, and in presence of external fields. In particular, we show direct evidence of antiparallel (parallel) alignment between the FM and the pinned (unpinned) uncompensated AFM moments in perpendicular exchange-coupled FM/AFM systems, and quantify directly the amount of pinned (unpinned) moments. In addition, we provide direct evidences at the nanometer scale and in external magnetic fields of their magnetization reversal behavior with element selectivity. The lateral resolution we realized so far is about $50 \mathrm{~nm}$, while we could image $1 \mathrm{ML}$ thick buried layers. This is a promising step toward imaging even thinner samples or systems with little magnetic contrast.

Future trends are focused on the experimental improvement, i.e., higher spatial resolution and magnetic sensitivity, but also should explore new scientific issues. For instance, a 
new concept for soft $\mathrm{x}$-ray holography imaging overcoming the restriction of a position-fixed and size-limited FOV of holography measurements has been recently introduced. ${ }^{26}$ In this case, all holography-optical elements are separated from the magnetic sample and are moveable with respect to the sample, allowing to explore different areas over the sample. Better spatial resolution can actually be achieved by using smaller reference holes with iterative phase retrieval methods ${ }^{10}$ and/or extended references by using the HERALDO (holography with extended reference by autocorrelation linear differential operation) concept. ${ }^{27}$ Nondestructive resonant magnetic scattering measurements using single pulses from a free electron laser source probe elementary magnetization dynamics in the femtosecond regime. ${ }^{28}$

Future scientific tasks will be related with both temperature, current-induced, time-resolved, and single-shot experiments performed in both perpendicular and in-plane magnetic anisotropy systems. For example, in-plane magnetic sensitivity could be achieved by combining extended reference apertures in off-normal transmission geometry experiments. These trends open a route toward a high spatiotemporal resolution which is needed to understand the microscopic origin of magnetization reversal in current and future magnetic complex systems.

\section{ACKNOWLEDGMENTS}

We acknowledge technical support by the ESRF staff R. Barrett, R. Homs-Regojo, T. Trenit, and G. Retout. A. B. acknowledges support through a Ramón y Cajal contract from the Spanish MICINN. This work was supported in part by the Spanish MICINN through Projects CSD2007-00010, and MAT2010-21822 and by Comunidad de Madrid through Project S2009/MAT-1726.

${ }^{1}$ Ultrathin Magnetic Structures, edited by J.A.C. Bland and B. Heinrich (Springer, Berlin, 2005), Vol. 3.

${ }^{2}$ W. Kuch, Magnetic Imaging, Lecture Notes in Physics (Springer, Berlin, 2006), Vol. 697, p. 275.

${ }^{3}$ H. A. Dürr, T. Eimüller, H.-J. Elmers, S. Eisebitt, M. Farle, W. Kuch, F. Matthes, M. Martins, H.-C. Mertins, P. M. Oppeneer, L. Plucinski, C. M. Schneider, H. Wende, W. Wurth, and H. Zabel, IEEE Trans. Magn. 45, 15 (2009).

${ }^{4}$ J. Miguel, J. Camarero, J. Vogel, J. Peters, N. B. Brookes, and J. Goedkoop, Appl. Surf. Sci. 254, 335 (2007).

${ }^{5}$ S. Eisebitt, J. Lüning, W. F. Schlotter, M. Lörgen, O. Hellwig, W. Eberhardt, and J. Stöhr, Nature (London) 432, 885 (2004).
${ }^{6}$ O. Hellwig, S. Eisebitt, W. Eberhardt, W. F. Schlotter, J. Lüning, and J. Stöhr, J. Appl. Phys. 99, 08H307 (2006).

${ }^{7}$ A. Scherz, W. F. Schlotter, K. Chen, R. Rick, J. Stöhr, J. Lüning, I. McNulty, Ch. Günther, F. Radu, W. Eberhardt, O. Hellwig, and S. Eisebitt, Phys. Rev. B 76, 214410 (2007).

${ }^{8}$ T. Hauet, C. M. Günther, B. Pfau, M. E. Schabes, J.-U. Thiele, R. L. Rick, P. Fischer, S. Eisebitt, and O. Hellwig, Phys. Rev. B 77, 184421 (2008).

${ }^{9}$ C. M. Günther, F. Radu, A. Menzel, S. Eisebitt, W. F. Schlotter, R. Rick, J. Lüning, and O. Hellwig, Appl. Phys. Lett. 93, 072505 (2008).

${ }^{10}$ S. Streit-Nierobisch, D. Stickler, C. Gutt, L.-M. Stadler, H. Stillrich, C. Menk, R. Frömter, C. Tieg, O. Leupold, H. P. Oepen, and G. Grübel, J. Appl. Phys. 106, 083909 (2009).

${ }^{11}$ C. Tieg, E. Jiménez, J. Camarero, J. Vogel, C. Arm, B. Rodmacq, E. Gautier, S. Auffret, B. Delaup, G. Gaudin, B. Dieny, and R. Miranda, Appl. Phys. Lett. 96, 072503 (2010).

${ }^{12}$ W. F. Schlotter, R. Rick, K. Chen, A. Scherz, J. Stöhr, J. Lüning, S. Eisebitt, Ch. Günther, W. Eberhardt, O. Hellwig, and I. McNulty, Appl. Phys. Lett. 89, 163112 (2006).

${ }^{13}$ W. H. Meiklejohn and C. P. Bean, Phys. Rev. 102, 1413 (1956).

${ }^{14} \mathrm{~F}$. Radu and H. Zabel, Springer Tracts in Modern Physics (Springer-Verlag Berlin 2007), Vol. 227, pp. 97-184.

${ }^{15}$ F. Garcia, J. Moritz, F. Ernult, S. Aufret, B. Rodmacq, B. Dieny, J. Camarero, Y. Pennec, S. Pizzini, and J. Vogel, IEEE Trans. Magn. 38, 2730 (2002).

${ }^{16}$ J. Camarero, J. Sort, A. Hoffmann, J. M. García-Martín, B. Dieny, R. Miranda, and J. Nogués, Phys. Rev. Lett. 95, 057204 (2005).

${ }^{17}$ E. Jiménez, J. Camarero, J. Sort, J. Nogués, A. Hoffmann, N. Mikuszeit, J. M. García-Martín, B. Dieny, and R. Miranda, Phys. Rev. B. 80, 014415 (2009).

${ }^{18}$ H. Ohldag, A. Scholl, F. Nolting, E. Arenholz, S. Maat, A. T. Young, M. Carey, and J. Stöhr, Phys. Rev. Lett. 91, 017203 (2003).

${ }^{19}$ J. Camarero, Y. Pennec, J. Vogel, S. Pizzini, M. Cartier, F. Fettar, F. Ernult, A. Tagliaferri, N. B. Brookes, and B. Dieny, Phys. Rev. B 67, 020413 (2003).

${ }^{20}$ J. Camarero, J. Miguel, J. Goedkoop, J. Vogel, F. Garcia, F. Romanens, S. Pizzini, N. B. Brookes, J. Sort, and B. Dieny, Appl. Phys. Lett. 89, 232507 (2006).

${ }^{21}$ R. Nakajima, J. Stöhr, and Y. U. Idzerda, Phys. Rev. B 59, 6421 (1999).

${ }^{22}$ M. Tsunoda, T. Nakamura, M. Naka, S. Yoshitaki, C. Mitsumata, and M. Takahashi, Appl. Phys. Lett. 89, 232507 (2006).

${ }^{23}$ S. Doi, N. Awaji, K. Nomura, T. Hirono, T. Nakamura, and H. Kimura, Appl. Phys. Lett. 94, 232504 (2009).

${ }^{24}$ I. Schmid, P. Kappenberger, O. Hellwig, M. J. Carey, E. E. Fullerton, and H. J. Hug, Europhys. Lett. 81, 17001 (2008).

${ }^{25}$ B. Kaplan and G. A. Gehring, J. Magn. Magn. Mater. 128, 111 (1993).

${ }^{26}$ D. Stickler, R. Frömter, H. Stillrich, C. Menk, C. Tieg, S. Streit-Nierobisch, M. Sprung, C. Gutt, L.-M. Stadler, O. Leupold, G. Grübel, and H. P. Oepen, Appl. Phys. Lett. 96, 042501 (2010).

${ }^{27}$ D. Zhu, M. Guizar-Sicairos, B. Wu, A. Scherz, Y. Acremann, T. Tyliszczak, P. Fischer, N. Friedenberger, K. Ollefs, M. Farle, J. R. Fienup, and J. Stöhr, Phys. Rev. Lett. 105, 043901 (2010).

${ }^{28}$ C. Gutt, S. Streit-Nierobisch, L.-M. Stadler, B. Pfau, C. M. Günther, R. Knnecke, R. Frömter, A. Kobs, D. Stickler, H. P. Oepen, R. R. Fäustlin, R. Treusch, J. Feldhaus, E. Weckert, I. A. Vartanyants, M. Grunze, A. Rosenhahn, T. Wilhein, S. Eisebitt, and G. Grübel, Phys. Rev. B 81, 100401 (2010). 